\title{
Acquired equivalence between stimuli trained in the same context
}

\author{
Mikael Molet • Holly C. Miller • Thomas R. Zentall
}

Published online: 5 April 2011

(C) Psychonomic Society, Inc. 2011

\begin{abstract}
The role of context was examined in human acquired equivalence. Participants were trained on two conditional discriminations. In the first conditional discrimination, if sample A1 was presented, choice of comparison $\mathrm{B} 1$, but not B2, was correct, and if sample A2 was presented, choice of comparison B2, but not B1, was correct. In the second conditional discrimination, if sample $\mathrm{X} 1$ was presented, choice of comparison $\mathrm{Y} 1$, but not $\mathrm{Y} 2$, was correct, and if sample $\mathrm{X} 2$ was presented, choice of comparison $\mathrm{Y} 2$, but not $\mathrm{Y} 1$, was correct. In each conditional discrimination, one of the conditional relations was trained in context 1 (e.g., $\mathrm{A} 1 \rightarrow \mathrm{B} 1$ and $\mathrm{X} 1 \rightarrow \mathrm{Y} 1$ ) and the other was trained in context 2 (i.e., $\mathrm{A} 2 \rightarrow \mathrm{B} 2$ and $\mathrm{X} 2 \rightarrow \mathrm{Y} 2$ ). On test trials, when conditional stimuli from the two conditional discriminations were interchanged (e.g., sample A1 was presented with comparisons $\mathrm{Y} 1$ and $\mathrm{Y} 2$ ) and were presented in a neutral context, positive transfer resulted. That is, in the absence of the training context, stimuli that shared a common context on different trials in training came to be treated as equivalent.
\end{abstract}

\section{Keywords Equivalence $\cdot$ Context $\cdot$ Human learning}

\author{
M. Molet \\ Université de Lille, Nord de France, \\ Lille, France \\ H. C. Miller • T. R. Zentall \\ University of Kentucky, \\ Pikeville, KY, USA \\ M. Molet $(\square)$ \\ Université de Lille, Nord de France, \\ Domaine universitaire du "Pont de Bois", \\ rue du Barreau, BP 60149 , \\ 59653 Villeneuve d'Ascq Cedex, France \\ e-mail: mikael.molet@univ-lille3.fr
}

Acquired equivalence refers to the phenomenon that when two different stimuli are associated with a common event, these stimuli can come to be regarded as equivalent. Thus, subsequent learning about one stimulus can be transferred to the other stimulus in the absence of direct training.

Urcuioli, Zentall, Jackson-Smith, and Steirn (1989) reinforced pigeons' choice of comparison W following presentation of sample A and sample B; choice of the alternative, comparison $\mathrm{X}$, was reinforced following presentation of two other samples, $\mathrm{C}$ and $\mathrm{D}$ (formally $\mathrm{A} \rightarrow \mathrm{W}$, $\mathrm{B} \rightarrow \mathrm{W}$ and $\mathrm{C} \rightarrow \mathrm{X}, \mathrm{D} \rightarrow \mathrm{X})$. The pigeons were then trained to associate one pair of those samples, $\mathrm{A}$ and $\mathrm{C}$, with new comparison stimuli $\mathrm{Y}$ and $\mathrm{Z}(\mathrm{A} \rightarrow \mathrm{Y}$ and $\mathrm{C} \rightarrow \mathrm{Z})$. Evidence for emergent stimulus relations was found when the remaining samples from original training (i.e., B and D) were then presented with the new comparisons ( $\mathrm{Y}$ and $\mathrm{Z}$ ). In the absence of any explicit training, most of the pigeons chose $\mathrm{Y}$ when the sample was $\mathrm{B}$ and chose $\mathrm{Z}$ when the sample was D (see also Bonardi, Rey, Richmond, \& Hall, 1993). Such evidence of transfer is the hallmark of an acquired equivalence between cues trained with common comparison stimuli. This kind of acquired equivalence has also been demonstrated in rats (Honey \& Hall, 1989; WardRobinson \& Hall, 1999) and humans (Hall, Mitchell, Graham, \& Lavis, 2003; Smyth, Barnes-Holmes, \& Barnes-Holmes, 2008).

Similar research has been conducted with rats, with stimuli that shared a common antecedent (Hall, Ray, \& Bonardi, 1993). In the first phase, each of two different auditory stimuli (A and B) was repeatedly preceded by a food pellet (food $\rightarrow$ A and food $\rightarrow B$ ), while a third auditory stimulus $C$ was not. Rats then received pairings of $A$ with an electrical shock ( $\rightarrow$ shock). In testing, Stimulus B evoked strong fear, as compared with stimulus $\mathrm{C}$, which had never been preceded by the food pellet (see also Johns \& Williams, 
1998; Sawa \& Nakajima, 2002). This acquired equivalence has also been reported with pigeons (Zentall et al., 1992; but see Urcuioli \& Zentall, 1993, for contrary evidence).

The explanation of acquired equivalence has typically been based on an associative chain mediated by the representation of the common event, the so-called representation-mediation hypothesis (Bonardi et al., 1993; Gluck \& Myers, 2001; Johns \& Williams, 1998; Meeter, Shohamy, \& Myers, 2009; Nosofsky, 1988; Sawa \& Nakajima, 2002; Smyth et al., 2008). The idea is that during initial training, the representations of stimuli paired with the same outcome or antecedent are modified to enhance generalization between them. This approach assumes that associative links or category assignments are stored in memory as a representation of the stimuli. In this way, at test, presentation of one stimulus evokes retrieval of stimulus representations from memory, and a response is given on the basis of the theoretical link or the category assignment stored in the retrieved representation. In Urcuioli et al. (1989), stimuli A and B were first paired with a common event $\mathrm{W}(\mathrm{A} \rightarrow \mathrm{W}, \mathrm{B} \rightarrow \mathrm{W})$, presumed to cause event $\mathrm{W}$ to be linked to or incorporated into memory representations of $\mathrm{A}$ and $\mathrm{B}$ (the same rationale is true for the samples $\mathrm{C}$ and $\mathrm{D}$ with the comparison $\mathrm{X}$ ). Then, when A was paired with a different event $\mathrm{Y}, \mathrm{Y}$ was linked to or incorporated into the representation of A. Thus, when B was presented, it was presumed to retrieve event $\mathrm{W}$, but event $\mathrm{W}$ should lead to retrieval of the representation of A, which in turn should lead to retrieval of Y. In this way, it is possible for an emergent relation to develop between B and $\mathrm{Y}$, as was observed by Urcuioli et al. (the same rationale can be used for the emergent relation between sample D and comparison Z). In Hall et al. (1993), stimuli A and B were first paired with a common event (food; in the backward direction: food $\rightarrow \mathrm{A}$, food $\rightarrow \mathrm{B}$ ), leading food to be linked to or incorporated into memory representations of $\mathrm{A}$ and $\mathrm{B}$. Then, when $\mathrm{A}$ was paired with a different event (shock), it was also linked to or incorporated in the representation of A. Thus, when B was presented, it would retrieve the food event, which should lead to the retrieval of a representation of $\mathrm{A}$, which in turn would lead to retrieval of shock. Thus, Hall et al. (1993) observed that presentation of B appeared to evoke a response of shock.

By extending the common element principle, one may ask whether stimuli that have shared a common context at different times can come to be treated as equivalent. However, currently, little is known about the contextual determinants of how an organism learns that two or more stimuli are equivalent when they are mapped onto the same element. The role of context has been investigated in a stimulus equivalence paradigm, but from a different perspective. Dibbets, Maes, and Vossen (2002) reported context dependency in a stimulus equivalence paradigm with humans. In this study, a shorter response latency was found when testing was conducted in the original context than when it was conducted in a different context (see also Bouton, 1993). A second study with humans indicated that the context served as an effective conditional stimulus to indicate to which stimulus class a stimulus belonged (Bush, Sidman, \& de Rose, 1989). However, the purpose of these studies was not to explore the role of the context in acquired equivalence but, rather, to show the generalization decrement that results from context change.

The present experiment was designed to investigate whether the context in which a conditional discrimination is acquired can serve to mediate an association between the sample from one conditional discrimination with the comparison from another. That is, can the context form the basis for an acquired equivalence set that can be demonstrated using a transfer-of-training design (see, e.g., Hall et al., 2003; Urcuioli et al., 1989) in the absence of the context itself? In our experiment, human participants were trained on one conditional discrimination that required them to learn to associate a sample with a comparison in context 1 (Cxt1) and another sample with the other comparison stimulus in a different context 2 (Cxt2). They were also trained on a second conditional discrimination for which correct choice of one comparison stimulus depended on the presence of one sample trained in Cxt1, whereas correct choice of the other comparison stimulus depended on the presence of a different sample trained in Cxt2. We then asked whether stimuli that shared a common context on different trials could come to be treated as equivalent. That is, in the absence of the training context, would there be positive transfer when conditional stimuli from the two conditional discriminations were interchanged?

\section{Method}

Participants

Sixteen undergraduate students (age, 18-23 years) at the University of Kentucky participated in this study in partial fulfillment of a psychology course requirement.

Apparatus and procedure

Participants performed the experiment using a Dell Latitude E540 with a monitor resolution of $1,024 \times 768$ pixels and a 32-bit color set in isolated cubicles (with a standard mouse). We used a matching-to-sample task that was programmed using Visual Basic 6 (Microsoft).

The stimuli for the two conditional discriminations consisted of symbols. All the stimuli were black and were approximately $5 \mathrm{~cm}$ in diameter. Each stimulus set was 
presented against one of the two background colors: soft blue or soft red. These background colors served as Cxt1 and $\mathrm{Cxt} 2$ and were incidental to the conditional discriminations (see Dibbets et al., 2002). The symbols ?,, and $\Omega$ were used as samples A1, A2, X1, and X2 (counterbalanced among participants). The symbols $\lambda$, $¥$, and $\ddagger$ were used as comparisons B1, B2, Y1, and Y2 (counterbalanced among participants; see Fig. 1).

A trial started when the participant clicked on the play button centered on the computer screen and presented on a soft grey background. The click on the play button changed the color of the computer screen to one of two different background colors (i.e., a soft red or a soft blue) that served as contextual cues and presented the sample stimulus (e.g., A1 or A2). The sample was above the play button. After an interval of $500 \mathrm{~ms}$, the comparison stimuli (e.g., B1 and B2) were presented simultaneously below the sample. After the participant clicked on the correct choice button (located below the comparison stimuli), the word "correct" appeared between the comparison stimuli; otherwise, the word
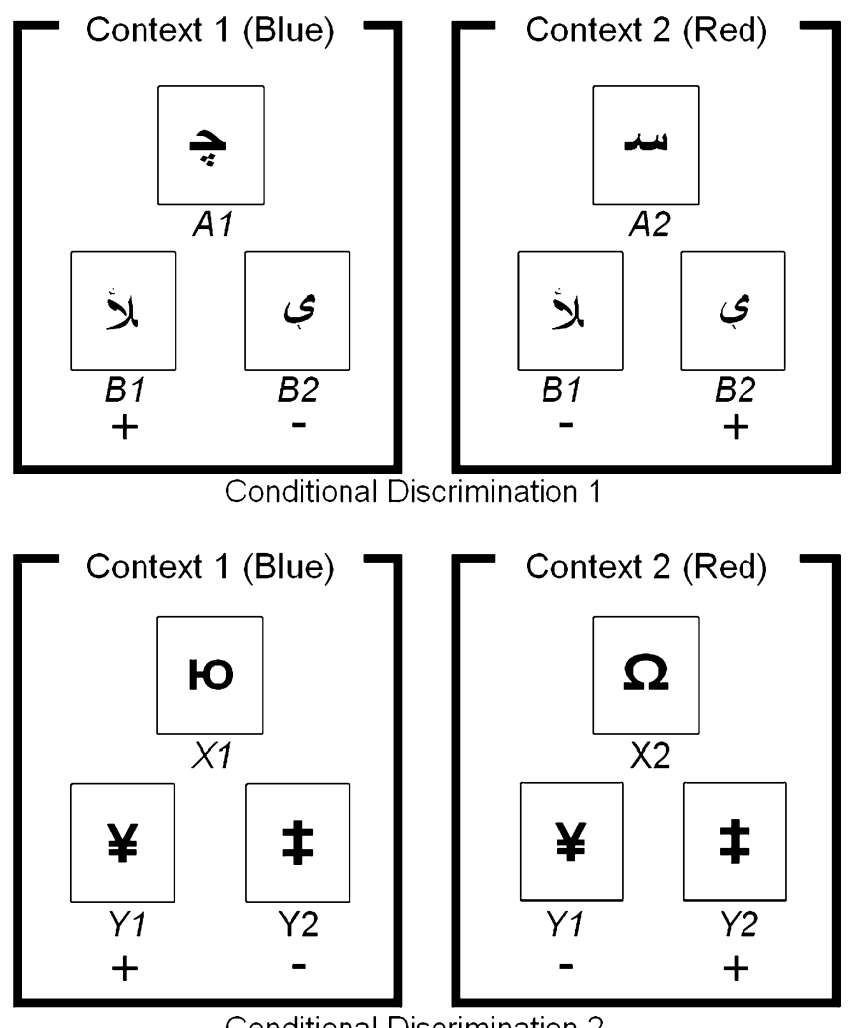

Fig. 1 Design of the conditional discriminations used in the experiment. Stimuli A1, A2, X1, and X2 were arbitrarily designated as samples, and stimuli B1, B2, Y1, and Y2 were arbitrarily designated as comparisons. "+" = correct comparison, "-“ incorrect comparison. Contexts 1 and 2 (Ctx 1 and $\mathrm{Ctx} 2$ ) were different background colors. Participants learned each conditional relationship in a specific context-formally, Ctx1: $\mathrm{A} 1 \rightarrow \mathrm{B} 1, \mathrm{Ctx} 2: \mathrm{A} 2 \rightarrow \mathrm{B} 2, \mathrm{Ctx} 1$ : $\mathrm{X} 1 \rightarrow \mathrm{Y} 1$, and $\mathrm{Ctx} 2: \mathrm{X} 2 \rightarrow \mathrm{Y} 2$. The conditions presented represent only one of the counterbalancing conditions "incorrect" was displayed. The feedback message remained visible for $1.0 \mathrm{~s}$. The color of the computer screen was then switched to the soft grey background color, and the stimuli disappeared from the screen.

The experiment was conducted in six phases. At the start of the experiment, each participant was placed in front of the computer screen. Instructions were presented in a white rectangular window on the screen.

In phase 1, the following instructions appeared on the computer screen:

You are about to play a game with a number of figures. When the game begins, this screen will first show you one figure at the top of the screen, followed by two figures at the bottom. It is your task to choose the correct figure at the bottom of the screen which matches the figure at the top of the screen. After you made your choice, the computer will tell you whether your choice was correct or incorrect. Later in the game, you will no longer be told whether your choice was correct or incorrect. You can still make the correct choices by paying attention from the start and by applying what you have already learned. Try to give as many correct responses as possible. When the experiment is over, a message will inform you that you can leave the room. If the instructions on the screen are unclear, please re-read them now. Otherwise, click below to start.

Phase 1: Conditional discrimination 1. Participants' choice of comparison B1 was correct following presentation of sample A1 in Ctx1, and choice of the alternative comparison $\mathrm{B} 2$ was correct following presentation of the other sample A2 in Ctx2-formally, Ctx1: A1 $\rightarrow$ B1 and Ctx2: $\mathrm{A} 2 \rightarrow \mathrm{B} 2$ (see Fig. 1, top). There was a minimum of 36 trials, 18 with each sample. Training trials were presented in random order with no more than 3 of the same sample trials presented consecutively. The left or right position of the comparisons was also randomized. Participants remained in phase 1 until they reached a criterion of $14 / 18$ correct trials involving each of the two samples. Participants who did not reach criterion in 36 trials were given an additional 36 trials of training.

The participants received the following instructions $2 \mathrm{~s}$ after completion of phase 1 :

Good! Now there will be a new problem with new figures. Once again, for each figure at the top, you will have to choose which figure at the bottom is correct. Try to give as many correct responses as possible. If the instructions on the screen are unclear, please re-read them now. Otherwise, click below to start. 
Phase 2: Conditional discrimination 2. The procedure was identical to that described in phase 1 , but with new samples and comparisons. Choosing comparison $\mathrm{Y} 1$ in the presence of sample $\mathrm{X} 1$ in Ctx1 was reinforced, whereas choosing comparison $\mathrm{Y} 2$ in the presence of sample $\mathrm{X} 2$ in $\mathrm{Ctx} 2$ was reinforced-formally, Ctx1: $\mathrm{X} 1 \rightarrow \mathrm{Y} 1$ and $\mathrm{Ctx} 2: \mathrm{X} 2 \rightarrow \mathrm{Y} 2$ (see Fig. 1, bottom).

The following instructions appeared on the screen $2 \mathrm{~s}$ after completion of phase 2 :

Now we are going to combine the two problems to see if you can perform them both at the same time. Try to give as many correct responses as possible. If the instructions on the screen are unclear, please re-read them now. Otherwise, click below to start.

Phase 3: Mixture of phasel and phase2. All the participants received 36 trials and were trained to a criterion of 7 out of 9 correct trials for each ofthe four samples.

The following instructions appeared on the screen $2 \mathrm{~s}$ after completion of phase 3 :

Now we are going to continue but at various times you will no longer receive feedback about whether your choice was correct or incorrect. You can still make the correct choices by applying what you have already learned. Try to give as many correct responses as possible. If the instructions on the screen are unclear, please re-read them now. Otherwise, click below to start.

Phase 4: Testing in a neutral context. Phase 4 consisted of 12 trials that were a continuation of phase 3 training (3 trials with feedback involving each of the four samples), randomly mixed among 24 test trials (6 trials without feedback involving each of the four samples in a neutral, grey background color context). These trials were introduced to determine the degree to which the samples controlled comparison choice in the absence of the context.

The following instructions appeared on the screen $2 \mathrm{~s}$ after completion of phase 4 :

Now we are going to combine the two problems againto see if you can still perform them at the same time. Try to give as many correct responses as possible. If the instructions on the screen are unclear, please re-read them now. Otherwise, click below to start.

Phase 5: Return to phase 3. The procedure was identical to that described in Phase 3.
Participants received the following instructions $2 \mathrm{~s}$ after completing phase 5:

Now we are going to continue but once again at various times you will no longer be given feedback as to whether your choice was correct or incorrect. You can still make the correct choices by applying what you have already learned. Try to give as many correct responses as possible. If the instructions on the screen are unclear, please re-read them now. Otherwise, click below to start.

Phase 6: Transfer test in neutral context. Phase 6 consisted of 12 trials that were a continuation of phase 5 training ( 3 trials involving each of the four samples, with feedback) and 24 transfer trials without feedback involving new combinations of samples and comparisons in the context of the neutral grey background (6 trials with each sample). A1 and A2 samples were followed by the simultaneous presentation of $\mathrm{Y} 1$ and $\mathrm{Y} 2$ comparisons, and $\mathrm{X} 1$ and $\mathrm{X} 2$ samples were followed by the simultaneous presentation of B1 and B2 comparisons (see Fig. 2). The purpose of the
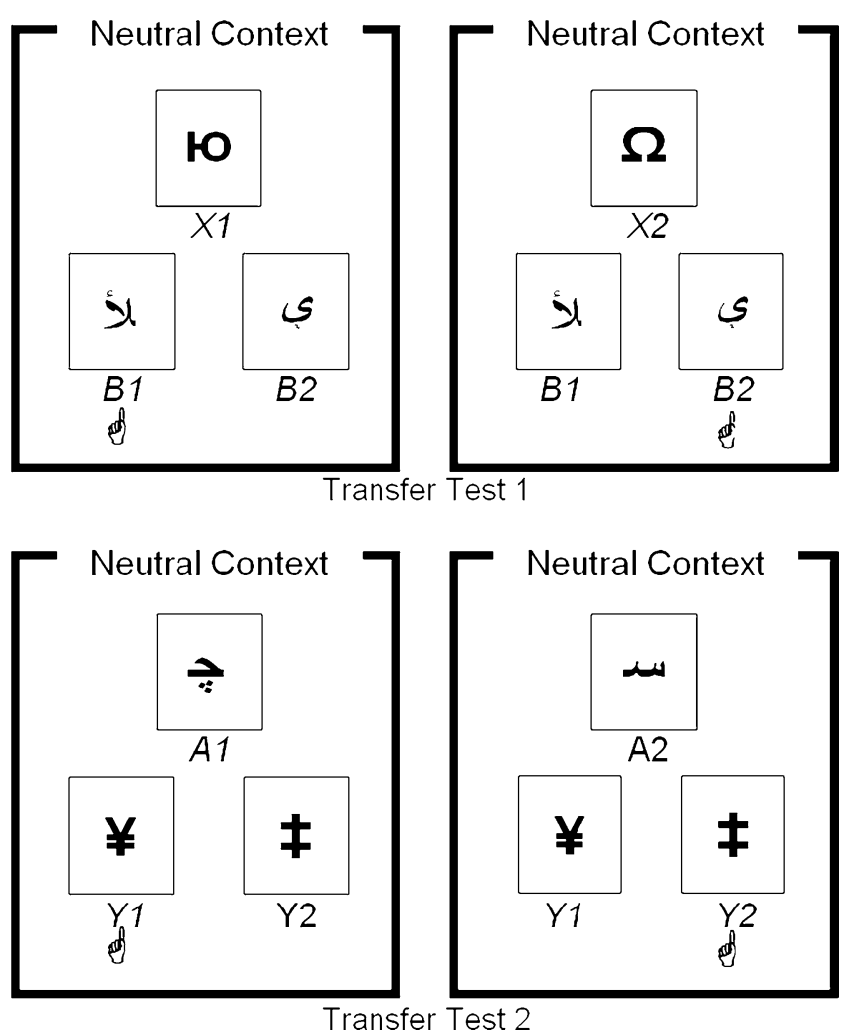

Fig. 2 Design of transfer trials in neutral context (soft grey background) where stimuli $\mathrm{A} 1$ and $\mathrm{X} 1$ were interchanged and stimuli $\mathrm{A} 2$ and $\mathrm{X} 2$ were interchanged. X1 and X2 samples were followed by simultaneous presentations of $\mathrm{B} 1$ and $\mathrm{B} 2$ comparisons, whereas $\mathrm{A} 1$ and $\mathrm{A} 2$ were followed by presentations of $\mathrm{Y} 1$ and $\mathrm{Y} 2$ comparisons. Choice based on context-mediated equivalence is indicated by " $\mathrm{d}$ " 
transfer trials was to test for the participants' use of the absent context as a mediator for choice of the comparison stimulus associated with the sample that had been presented in the same colored-background context as the presented sample.

After completing the experiment, the participants received the following instructions:

Thank you for participating in this study. You can leave this room and look for the experimenter.

\section{Results and discussion}

All but 2 of the participants acquired each of the conditional discriminations within the first 36 training trials, and errors in phases 3 and 5 were few. In phase 4, the proportion of correct responses for each conditional relation tested without feedback and in a neutral context was above $97 \%$, demonstrating that the choice of the comparisons did not depend on the context in which they were acquired.

The critical results are the transfer data from phase 6 neutral-context trials in which the participants had to choose comparisons when conditional stimuli from the two conditional discriminations were interchanged. Overall, participants chose the comparison that had been correct in the same context as the sample on $75.8 \%$ of the test trials. On X1 transfer trials, the mean choice of the B1 comparison was $78 \%(S D=7.09)$; on $\mathrm{X} 2$ transfer trials, the mean choice of the B2 comparison was $76 \%(S D=$ 8.73); on A1 transfer trials, the mean choice of the Y1 comparison was $73 \%(S D=7.74)$; and on A2 transfer trials, the mean choice of the Y2 comparison was $76 \%$. $(S D=8.46)$. These scores were each significantly different from chance $(50 \%), t(15)=3.96,2.90,2.96$, and 3.07, respectively, all $p$ values $<.05$.

Of the 4 participants who did not show good transfer in phase 6, 2 made errors in training comparable to those the majority of the participants who showed good transfer, the 2 others needed an additional block of trials to reach criterion in phase 1 .

The results of this experiment demonstrate that emergent relations can develop between stimuli that are presented in the same context but at different times, as indicated by a transfer-of-training test in the absence of that common context. The emergent relations appear to be mediated by the activation of a representation of the common context by the sample presented, and the context, in turn, appears to activate a representation of either the other sample that was presented in that context or, more directly, the correct comparison associated with that sample. More specifically, presentation of sample A1 would retrieve a representation of Ctx1, which would, in turn, lead to retrieval of a representation of sample $\mathrm{X} 1$ or comparison $\mathrm{Y} 1$ (as well as comparison B1) and choice of Y1. Similarly, presentation of sample $\mathrm{X} 1$ would retrieve a representation of $\mathrm{Ctx} 1$ that would, in turn, lead to retrieval of a representation of sample A1 or comparison B1 and choice of B1. Similar mediation by way of Cxt2 would lead to choice of comparison Y2 upon presentation of sample A2 and to comparison B2 upon presentation of sample X2 (see Fig. 3).

In principle, presentation of a sample could lead to retrieval of the other sample that was presented in same context, and that other sample would lead to retrieval of the correct comparison. Alternatively, presentation of a sample could lead directly to the retrieval of the untrained comparison. In either case, transfer to the untrained comparison requires a context-mediated account.

The immediate implication of the results of the present experiment is that they extend our knowledge of the sort of training that is capable of producing generalization between stimuli that have been trained in the same way. As was noted earlier, humans and other animals may treat two perceptually different cues alike, if the cues have been individually paired with a common antecedent or a common consequence (e.g., Hall et al., 1993; Hall et al., 2003). Demonstration of contextual acquired equivalence gives further support for the generality of stimulus equivalence effects and reinforces the hypothesis that a common representation of the context can mediate equivalence learning.
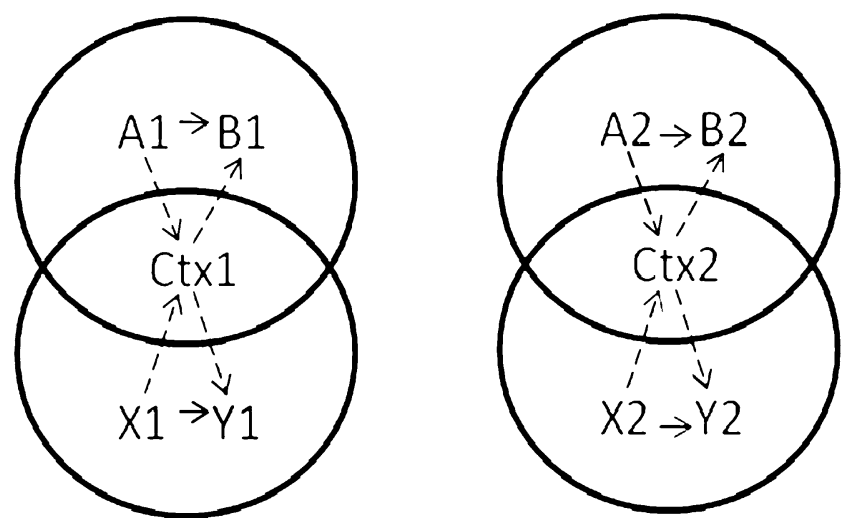

Fig. 3 A potential model for the development of emergent relations between samples and comparisons not presented together in training. Solid arrows represent trained associations. Dashed arrows represent presumed emergent relations, with context mediating the association between the sample from one conditional discrimination and the comparison from a second conditional discrimination 
Author Note Mikael Molet was supported by a research grant from the Université de Lille to work in TRZ's laboratory at the University of Kentucky. The research was supported by National Institute of Child Health and Development Grant HD060996 to the second author. We thank Peter Urcuioli for discussions involving the use of context as a mediator of equivalence.

\section{References}

Bonardi, C., Rey, V., Richmond, M., \& Hall, G. (1993). Acquired equivalence of cues in pigeon autoshaping: Effects of training with common consequences and with common antecedents. Animal Learning \& Behavior, 21, 369-376.

Bouton, M. E. (1993). Context, time, and memory retrieval in the interference paradigms of Pavlovian learning. Psychological Bulletin, 114, 80-99.

Bush, K. M., Sidman, M., \& de Rose, T. (1989). Contextual control of emergent equivalence relations. Journal of the Experimental Analysis of Behavior, 51, 29-45.

Dibbets, P., Maes, J. H. R., \& Vossen, J. M. H. (2002). Contextual dependencies in a stimulus equivalence paradigm. The Quarterly Journal of Experimental Psychology, 55B, 97-119.

Gluck, M. A., \& Myers, C. E. (2001). Gateway to memory: An introduction to neural network modeling of the hippocampus and learning. Cambridge: MIT Press.

Hall, G., Ray, E., \& Bonardi, C. (1993). Acquired equivalence between cues trained with a common antecedent. Journal of Experimental Psychology. Animal Behavior Processes, 19, 391-399.

Hall, G., Mitchell, C., Graham, S., \& Lavis, Y. (2003). Acquired equivalence and distinctiveness in human discrimination learning: Evidence for associative mediation. Journal of Experimental Psychology. General, 132, 266-276.
Honey, R. C., \& Hall, G. (1989). The acquired equivalence and distinctiveness of cues. Journal of Experimental Psychology. Animal Behavior Processes, 15, 338-346.

Johns, K. W., \& Williams, D. A. (1998). Acquired equivalence learning with antecedent and consequent unconditioned stimuli. Journal of Experimental Psychology. Animal Behavior Processes, 24, 3-14.

Meeter, M., Shohamy, D., \& Myers, C. E. (2009). Acquired equivalence changes stimulus representation. Journal of the Experimental Analysis of Behavior, 91, 127-141.

Nosofsky, R. M. (1988). Exemplar-based accounts of relations between classification, recognition and typicality. Journal of Experimental Psychology. Learning, Memory, and Cognition, 14, 700-708.

Sawa, K., \& Nakajima, S. (2002). Acquired equivalence of flavour cues with a common antecedent in rats. Behavioural Processes, 57, 1-6.

Smyth, S., Barnes-Holmes, D., \& Barnes-Holmes, Y. (2008). Acquired equivalence in human discrimination learning: The role of propositional knowledge. Journal of Experimental Psychology. Animal Behavior Processes, 34, 167-177.

Urcuioli, P. J., \& Zentall, T. R. (1993). A test of comparison-stimulus substitutability following one-to-many matching by pigeons. Psychological Reports, 43, 745-759.

Urcuioli, P. J., Zentall, T. R., Jackson-Smith, P., \& Steirn, J. N. (1989). Evidence for common coding in many-to-one matching: Retention, intertribal interference, and transfer. Journal of Experimental Psychology. Animal Behavior Processes, 15, 264-273.

Ward-Robinson, J., \& Hall, G. (1999). Backward sensory preconditioning. Journal of Experimental Psychology. Animal Behavior Processes, 22, 395-404.

Zentall, T. R., Sherburne, L. M., Steirn, J. N., Randall, C. K., Roper, K. L., \& Urcuioli, P. J. (1992). Common coding in pigeons: Partial versus total reversals of one-to-many conditional discriminations. Animal Learning \& Behavior, 20, 373-381. 